\title{
Liver Radiation Injury
}

National Cancer Institute

\section{Source}

National Cancer Institute. Liver Radiation Injury. NCI Thesaurus. Code C161519.

Radiation injury of the liver. 